\title{
Managing Migraine via Telemedicine: Clinical Effectiveness and Process Implications
}

\author{
Balaraman Rajan \\ California State University East Bay \\ balaraman.rajan@csueastbay.edu
}

\author{
Abraham Seidmann \\ University of Rochester \\ avi.seidmann@simon.rochester.edu
}

\author{
Deborah Friedman \\ UT Southwestern \\ deborah.friedman@utsouthwestern.edu
}

\begin{abstract}
Telemedicine has been proved to be effective across several different medical fields. It increases access to patients and reduces travel burden. In the context of an ongoing pilot study of telemedicine for individuals with migraine, we completed in-person baseline assessments and follow-up visits via telemedicine to test the hypothesis that follow-up care delivered by telemedicine is at least as effective as with in-office visits. We then investigate ways in which telemedicine could add economic value to patients through convenience and better compliance, and benefit specialists through efficient clinical pathway.
\end{abstract}

\section{Introduction}

Migraine is a chronic condition due to a neurovascular disorder that causes severe headaches and other neurologic symptoms. It can be debilitating and can last for a few hours or even days, if left untreated [6]. It is the third most common disease in the world and seventh in terms of specific causes of disability globally [18]. People suffering from Migraine may require complete bed rest and may have to miss school or work and may not be able to perform household chores or participate in leisure activities [12]. Hence it could severely disrupt a person's daily activities.

Even though there are several guidelines and treatment regimens available, many eligible individuals do not receive proper care [2,3]. Access to specialist, proper diagnosis, and following the treatment regimen are all important aspects of managing this disabling condition [13].

Telemedicine is the exchange of medical information via electronic communication to improve patient health [9]. Hersh et al. report how various psychiatric and neurological assessments have been administered effectively via interactive videoconferencing for medicare patients [8]. Interactive video conferencing allows a physician and a patient to communicate with each other from distant sites while simultaneously viewing and listening to each other. Thus, telemedicine helps bring medical specialists to remote patients who would not otherwise have access to such high quality and condition-specific care.

It has already been used for pediatrics, psychiatry, movement disorders, neurological disorders like Alzheimer's disease and epilepsy, dermatological disorders, and such chronic disorders as diabetes; see [8], [5], for various applications, and [15] for reviews of telemedicine's cost-effectiveness.

Kennedy et al. mention that when local physicians are limited by what they could potentially do, teleradiology has brought quality and timeliness and not only aids the local physicians but also equips them with the latest technology [11]. Telemedicine has also been seen as a means to increase access to specialty care for many individuals, especially in rural areas [1]. A review of evidence for the benefits of telemedicine has been presented by Hailey et al. [7].

Telemedicine has also been used by primary care physicians to consult specialists, avoiding expensive referrals in many cases [10]. It has been used to help nurses and physicians to even remotely monitor patients in multiple, scattered intensive care units [4].

On the economic and strategic benefits of telemedicine, Rajan et al. explore the impact of telemedicine on patient choices, hospital market shares and volume mix, and the role of local community hospitals. They show how telemedicine could increase access to patients and increase the market share of hospitals [16]. Meyer and Paré discuss the challenges and benefits of implementation [14].

Telemedicine and some of the latest information technology could well be used by patients to visit their specialist from their home. Even a simple webbased video conferencing facility (such as Skype) could substitute certain non-critical in-person visits. Telemedicine therefore could potentially act as a gateway to specialists. The convenience of 
telemedicine visits will also help patients keep up their appointments and hence help in care continuity.

Medical specialists for managing chronic conditions (such as Migraine) typically face patients coming from long distances and patients who are in different stages of their chronic condition. When dealing with such heterogeneous mix of patients, specialists need to make some strategic choices including number of appointments to be given in a day and the patient pool (panel size) to be maintained by them. Rajan et al. explore this strategic interaction between the patients and the specialist [17].

Migraine patients are followed up periodically, several times a year. Their visit frequency varies a fair amount, depending on their personal needs and conditions. While most of the patients can drive, they typically keep a full time job that could require unplanned travel to locations far away from the clinic. Patients, therefore, are told to book only one appointment at a time.

The feasibility of telemedicine varies from case to case and also from visit to visit. Thus some patients may be required to visit the office in person for further physical, or interventional testing, or simply for personal support. Other times, they could be managed through virtual (telemedicine) visits.

The migraine clinic is very busy, and to maintain a reasonable service level for patients in need - the attending MDs only serve a limited group (panel) of patients at a time.

In the context of an ongoing pilot study of telemedicine for individuals with migraine, we test the hypothesis that follow-up care delivered by telemedicine is as effective as with in-office visits. We then investigate ways in which telemedicine could add economic value to patients through convenience and better compliance, and benefit specialists through a higher productivity.

\section{Study Methodology}

The study was approved by the Institutional Review Boards at the University of Texas Southwestern Medical Center and the University of Rochester. Potential patients were first assessed in person for their current condition. After the initial (baseline) office visit, patients with a diagnosis of migraine were invited to be part of the telemedicine study. The patients who consented were then officially recruited for the study.

Enrolled patients were randomized to have follow-up visits either in person or using telemedicine on the following visit schedule: 4-6 weeks, 3 months, 6 months, 9 months and 12 months after their respective baseline visit. All participants were asked to complete questionnaires (through survey monkey) a few days before and immediately after each visit. All visits were conducted in a similar fashion.

Feasibility was measured by the percentage of visits completed as scheduled. We use Migraine Disability Assessment (MIDAS) scores to measure the disability caused by migraine. Improvement in MIDAS scores over the period of the study was used to measure the clinical effectiveness [2].

The travel and associated time costs were obtained from the surveys based on details regarding distance, and time spent for the appointment, travel and waiting. Patients also completed an overall satisfaction survey and were interviewed via telephone at the conclusion of the study for qualitative assessments.

We had 42 patients enrolled in the study out of whom 20 were randomized to the in-person arm and 22 patients were randomized to the telemedicine arm. Most of the patients had more than a basic exposure to internet with some even having attended video conferences.

Two patients declined participation at their initial visit, preferring in-person care. Twenty patients have completed the study. All twenty two patients assigned to telemedicine are being managed and followed via telemedicine without issue. Three of the 22 patients assigned to in-person care withdrew from the study citing travel as a factor. Thus far, 77 telemedicine visits were completed as scheduled,

\section{Initial Results and Insights}

As seen from Figure 1, most patients found telemedicine visits to be very convenient as opposed to the experience by patients undergoing in-person visits. This was expected given the travel requirement for some of the patients (for example, one patient had to travel 300 miles to see the specialist).

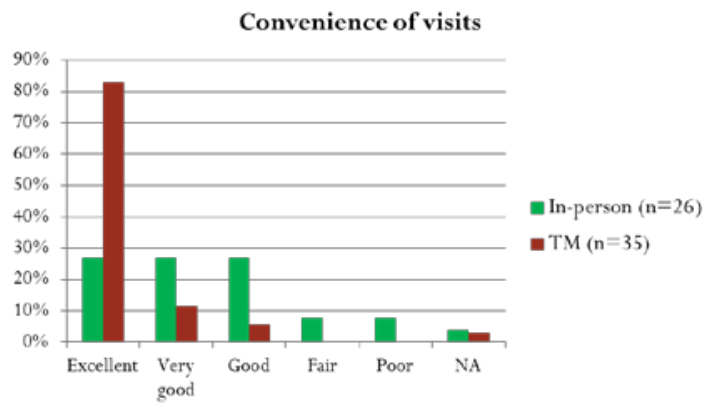

Figure 1: Comparison of patient experience with in-person vs. telemedicine visits 
Participants spent on average 3 hours and 8 minutes $(\mathrm{n}=46)$ for an in-person visit vs. 35 minutes $(n=59)$ for a telemedicine visit. (Note: the number (n) varies in each of the figures depending on the number of patients who had completed the respective surveys. Also, since this is still an ongoing study, we have different patients at different stages of the study now). The savings in time eliminated the need to take work days off for their care.

As seen from Figure 2, telemedicine visits took only a few minutes (mostly between 30 minutes and 1 hour) and hence there was less of a need to take time off from work. In fact, some patients attended their visits from their workplace.

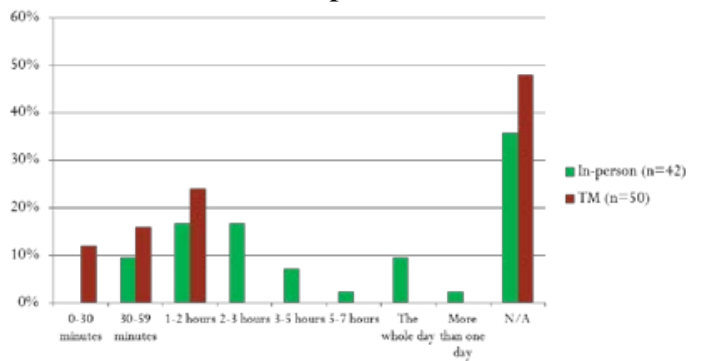

Figure 2: Comparison of Time off from work for in-person vs. telemedicine visits

5 out of 46 visits required the patient seen in person to take at least one day off vs none of 59 visits for patients seen via telemedicine. 17 out of 46 visits required the patient seen in person to take at least two hours off vs 3 out of 59 visits for telemedicine. The benefit could also be directly linked to Figure 1, based on the convenience of the visits.

As seen from Figures 3 and 4, most of the patients in the in-person arm considered telemedicine visits to be broadly the same as in-person visits but many patients in the telemedicine arm even considered telemedicine visits to be of superior nature to inperson visits.

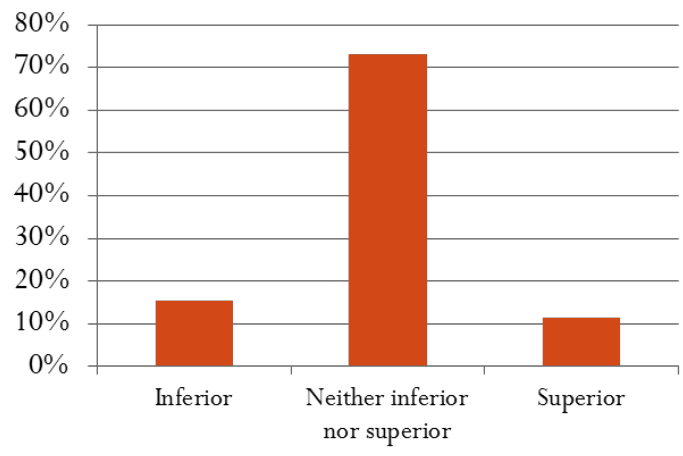

Figure 3: Perception of Telemedicine by patients in the in-person arm
From Figure 5, disability improved both patients seen through both telemedicine and in-person visits. The MIDAS scores in fact, fell by a bigger magnitude for the telemedicine arm than the inperson arm. But this was also due to the baseline MIDAS scores being lower for the in-person arm than the telemedicine arm (38.61 vs. 62.52).

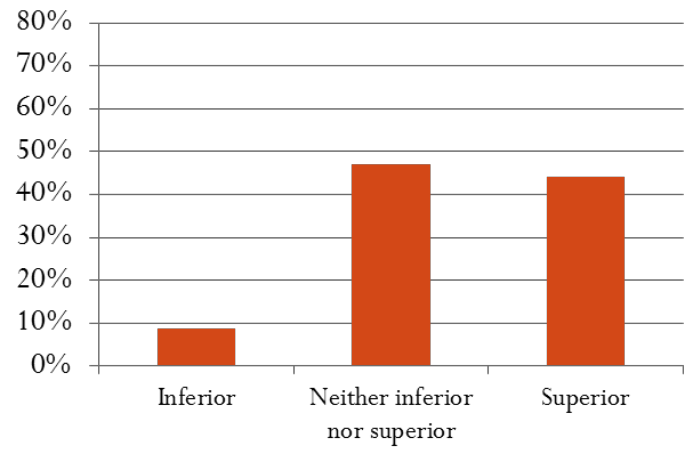

Figure 4: Perception of Telemedicine by patients in the telemedicine arm

From the surveys and the interviews some of the advantages of telemedicine were very clear. Patients appreciated the fact that there was no travel involved with telemedicine visits and there was no need to take time off from work. More than the travel cost and the associated time, the burden was also lifted from these patients who were suffering from a disabling condition. Travel even compounded their suffering sometimes.

Patients also felt relaxed and not rushed in a home setting. They considered telemedicine visits to be more efficient with less non-doctor interactions and repetitive questions from clinic staff. List of other significant comments from our study participants can be found in Appendix 6.1.

Interestingly, some patients considered telemedicine visits to be more personable (the idea that a doctor is "coming" to see a patient like in a house call) while some considered the virtual visits to be less personable.

Some patients considered the telemedicine visits as a barrier to communication. There were also concerns about telemedicine visits not being a feasible means to perform certain critical exam (such as administering an injection). List of other significant comments from our study participants can be found in Appendix 6.2. 


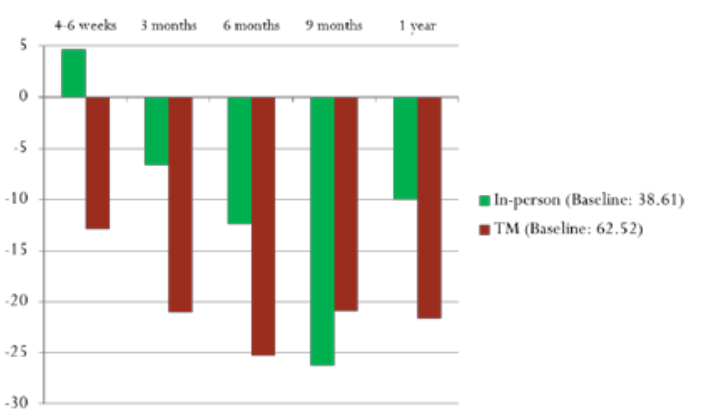

Figure 5: Comparison of change in MIDAS scores for in-person vs. telemedicine visits

\section{Process Impact of Telemedicine}

To test the effect of telemedicine, we ran a simple regression model with improvement in MIDAS score as the dependent variable (Imp). We used the baseline MIDAS scores (BM), type of visits (TV), and Internet familiarity (Int) as independent variables. TV is a dummy variable taking a value of 1 if it the patient is in the telemedicine arm and 0 if the patient is in the in-person arm.

$$
\operatorname{Imp}=\beta_{0}+\beta_{1} B M+\beta_{2} T V+\beta_{3} \operatorname{Int}
$$

We hypothesize that the improvement in MIDAS scores will not be dependent on the type of the visit $\left(\beta_{2}=0\right)$, after controlling for the baseline MIDAS scores. Our initial results (Table 1 ) do support our hypothesis and the only variable that was significant was BM, the baseline MIDAS scores. This will lead us to the result that in terms of clinical effectiveness seeing the patients via telemedicine or in person will not make a difference.

\begin{tabular}{|l|r|r|r|r|}
\hline & Coefficients & \multicolumn{1}{c|}{ SE } & t Stat & P-value \\
\hline Intercept & 15.05 & 11.13 & 1.35 & 0.20 \\
\hline TV & -5.61 & 10.59 & -0.53 & 0.60 \\
\hline BM & 0.36 & 0.09 & 4.09 & 0.00 \\
\hline Int & 0.45 & 2.80 & 0.16 & 0.88 \\
\hline
\end{tabular}

Table 1: Regression results

But, interestingly, the time spent by the specialist with the patient during the visit varied significantly between the two kinds of visits. While telemedicine visits took on average 23 minutes, the in-person visits took about 33 minutes. This renders empirical evidence to the analytical work by Rajan et al. [17] who prove that telemedicine visits will make the visit durations shorter.
Since most visits to a specialist involve a great deal of time from the patient side, specialists tend to compensate for this travel burden by spending more time with the patients. This is in spite of the fact that as the specialists spend more time with each patient, the waiting time for other patients increases.

With the introduction of telemedicine, we find that, the utilization of a revenue-maximizing specialist goes up, service rate increases and he sees more patients and thus, gets closer to a welfaremaximizing specialist.

\section{Summary and Conclusion}

Telemedicine is a feasible means of conducting follow-up patient evaluations for individuals with migraine. Thus far, clinical effectiveness of each method seems to be similar for both groups, on average. Based on post-visit surveys, patients are highly satisfied with care delivered via telemedicine.

On the benefits side, first and foremost, telemedicine increases access to specialists. It also adds economic value to patients by reducing travel costs and more importantly eliminating the need to take time off from work. It also gives patients great flexibility so that there are able to keep up their appointments.

For the specialists, the visit times get shorter and hence telemedicine visits are more efficient. Though the utilization increases for the specialists, with more patients seeking treatment, revenue also increases with telemedicine. The specialists could also focus on higher end tasks such as interventional office procedures and delegate the rest for telemedicine visits. Thus, telemedicine could also lead to a more efficient clinical pathway. The study is ongoing and further analyses are underway.

\section{References}

[1] Bashshur RL, et al. Telemedicine: a new health care delivery system. Annual Review of Public Health. 2000 May;21(1):613-37.

[2] Bigal, Marcelo E., et al. Assessment of migraine disability using the migraine disability assessment (MIDAS) questionnaire: a comparison of chronic migraine with episodic migraine. Headache: The Journal of Head and Face Pain 43.4 2003: 336-342.

[3] Bigal ME, et al. The acute treatment of episodic and chronic migraine in the USA. Cephalalgia. 2009 Aug 1;29(8):891-7. 
[4] Cummings J, et al. Intensive care unit telemedicine: review and consensus recommendations. American Journal of Medical Quality. 2007 Jul 1;22(4):239-50.

[5] Dorsey, E.R., et al. Randomized, controlled trial of "virtual housecalls" for Parkinson disease. The Journal of the American Medical Association (JAMA) Neurology. 2013. 70(5) 565-70.

[6] Goadsby PJ, Lipton RB, Ferrari MD. Migraine-current understanding and treatment. New England Journal of Medicine. 2002 Jan 24;346(4):257-70.

[7] Hailey D, Roine R, Ohinmaa A. Systematic review of evidence for the benefits of telemedicine. Journal of telemedicine and telecare. 2002 Mar 1;8(suppl 1):1-7.

[8] Hersh, W.R., et al. Telemedicine for the Medicare population: Update. AHRQuality 2006. 131 1-41.

[9] http://www.americantelemed.org/abouttelemedicine/what-is-telemedicine

[10] Keely E, Liddy C, Afkham A. Utilization, benefits, and impact of an e-consultation service across diverse specialties and primary care providers. Telemedicine and eHealth. 2013 Oct 1;19(10):733-8.

[11] Kennedy S, et al. The effect of teleradiology on time to interpretation for CT pulmonary angiographic studies. Journal of the American College of Radiology. 2009 Mar 31;6(3):180-9.

[12] Lipton RB, et al. Prevalence and burden of migraine in the United States: Data from the American Migraine Study II. Headache. 2001;41:646-657.

[13] Lipton RB, et al. Barriers to the diagnosis and treatment of migraine: effects of sex, income, and headache features. Headache: The Journal of Head and Face Pain. 2013 Jan 1;53(1):81-92.

[14] Meyer J, Paré G. Telepathology Implementation Challenges and Benefits: A Scoping Review. InSystem Sciences (HICSS), 2014 47th Hawaii International Conference on 2014 Jan 6 2838-2847. IEEE.

[15] Mistry, H. Systematic review of studies of the cost-e ectiveness of telemedicine and telecare. Changes in the economic evidence over twenty years. Journal of telemedicine and telecare 2012 1(18) 1-6.

[16] Rajan B, Seidmann A, Dorsey ER. The competitive business impact of using telemedicine for the treatment of patients with chronic conditions. Journal of Management Information Systems. 2013 Oct 1;30(2):127-58.

[17] Rajan B., Seidmann A., and Tezcan T. Service systems with heterogeneous customers: Investigating the effect of telemedicine on patient care. Working Paper
[18] Steiner TJ, Stovner LJ, Birbeck GL. Migraine: the seventh disabler. Headache: The Journal of Head and Face Pain. 2013 Feb 1;53(2):227-9.

\section{Appendix}

6.1 Patient comments - Positive "I spend more time in the car than with the Dr." "I would hope that telemedicine would provide more access to a doctor for those who were unable to see their doctor in person. I would like more access to best health care."

"No travel to downtown, no toll fees, and no long walk from the parking garage to the office." "little interruption to normal schedule"

"I don't have to take off the entire day, drive 120 miles, fight the traffic, the horrible parking situation, eating out expensive."

"I like not having to rush to get to a doctors appointment and then waiting for them.”

"I would LOVE not having the hour + drive. (Usually ends up causing a migraine, which is exactly what I'm there to prevent!)"

"...in fact it was even better because I was even more relaxed being at home..”

"I do feel it is more personable. There is no wait time to see Dr. Friedman, and no repetitive questions from her staff.”

"I was able to do it from my office computer without leaving work."

"perfect for the busy schedule of UT employee mixed with the busy schedule of UT physician"

"It was better than most visits I've had in person with a doctor.”

6.2 Patient comments - Concerns

"I would not like to put any communication barriers between myself and my doctor, including a camera or distance."

"I do not get a real patient visit where the doctor reviews all my systems to see if I am getting better, the worse, or the same. Trying to provide good patient care over the Internet is superficial,"

"I think the doctor listens more carefully when I have an office visit, and I feel like I am able to better communicate my personal medical issues to the doctor."

"It may seem less personal."

"I would miss shaking hands with my doctor." 
"Lack of blood pressure check, etc prior to potentially starting a new medication"

"inability to be examined if needed."

"Intimidation"

"I didn't like that I couldn't get medicine right away if I need it."

"I would feel a little self-conscious about the status of house behind and around me. I have 2 kids at home ..” 